\title{
Ratio of lymph node aspiration washout tyroglobulin levels to whole blood and serum washout tyroglobulin levels in patients with differentiated thyroid carcinoma: A new approach for standardization
}

of measurements and increased accuracy

\section{Cevdet Aydin' ${ }^{1}$ Didem Ozdemir, ${ }^{1}$ Muhammed Sacikara ${ }^{1}$, Sefika Burcak Polat ${ }^{1}$, Aylin Kilic Yazgan², Seyda Turkolmez ${ }^{3}$, Eda Demir Onal ${ }^{1}$, Reyhan Ersoy ${ }^{1}$, Bekir Cakir ${ }^{1}$}

${ }^{1}$ Ankara Yildirim Beyazit University, School of Medicine, Ataturk Education and Research Hospital, Department of Endocrinology and Metabolism, Ankara, Turkiye

${ }^{2}$ Ankara Yildirim Beyazit University, School of Medicine, Ataturk Education and Research Hospital, Department of Pathology, Ankara, Turkiye

${ }^{3}$ Ankara Yildirim Beyazit University, School of Medicine, Ataturk Education and Research Hospital, Department of Nuclear Medicine, Ankara, Turkiye

\section{Introduction}

High values of fine needle aspiration washout tyroglobulin (FNA-Tg) are diagnostic for metastatic lesions of thyroid cancer. However, there is not a consensus on cut-off for high Tg level. The most important confounding factor for FNA-Tg is inability to calculate Tg per unit volume because residual aspiration material in the needle can not be known exactly. Another problem is possible contamination of whole blood during lymph node aspiratiom. In this study, we aimed to determine a more accurate and standardized parameter for FNA-Tg.

\section{Description of Methods:}

Ultrasonographically suspicious 155 lymph nodes in 120 patients with histopathologically confirmed differentiated thyroid cancer and suspicious for malignancy/malignant thyroid nodule FNA result in cytology were evaluated. Tg was measured in samples obtained by aspiration (FNA) and non-aspiration (FNNA) biopsy. The highest value obtained by these methods from the same lymph node was defined as FNB-Tg. Simultaneous venous blood sample was taken. $\mathrm{Tg}$ levels were measured from whole blood and serum washouts which were obtained by using syringes and needles identical to ones used for lymph node biopsy (Figure 1).

\section{Results:}

Data of 16 lesions in 14 patients who underwent lymph node dissection were analyzed. FNNA-Tg, FNB-Tg/whole blood washout-Tg, FNB-Tg/serum washout-Tg, FNB$\mathrm{Tg} /$ serum $\mathrm{Tg}$ were significantly higher in malignant lymph nodes compared to benign ones. Areas under the ROC curve for FNNA-Tg, FNNA-Tg/whole blood washout-Tg, FNNA-Tg/serum washout-Tg were statistically significant for the discrimination of benign and malignant lymph nodes (Table 1). Best cut-off values according to FNNA-Tg and FNB-Tg were 15.86 and $31.8 \mathrm{ng} / \mathrm{ml}$, respectively. Among ratios, best cut off values to discriminate benign and malignant lymph nodes were 5.44 for FNB-Tg/whole blood washout-Tg and 3.95 for FNB-Tg/serum washout-Tg.
Figure 1: Sampling methods for thyroglobulin measurement A) From lymph node

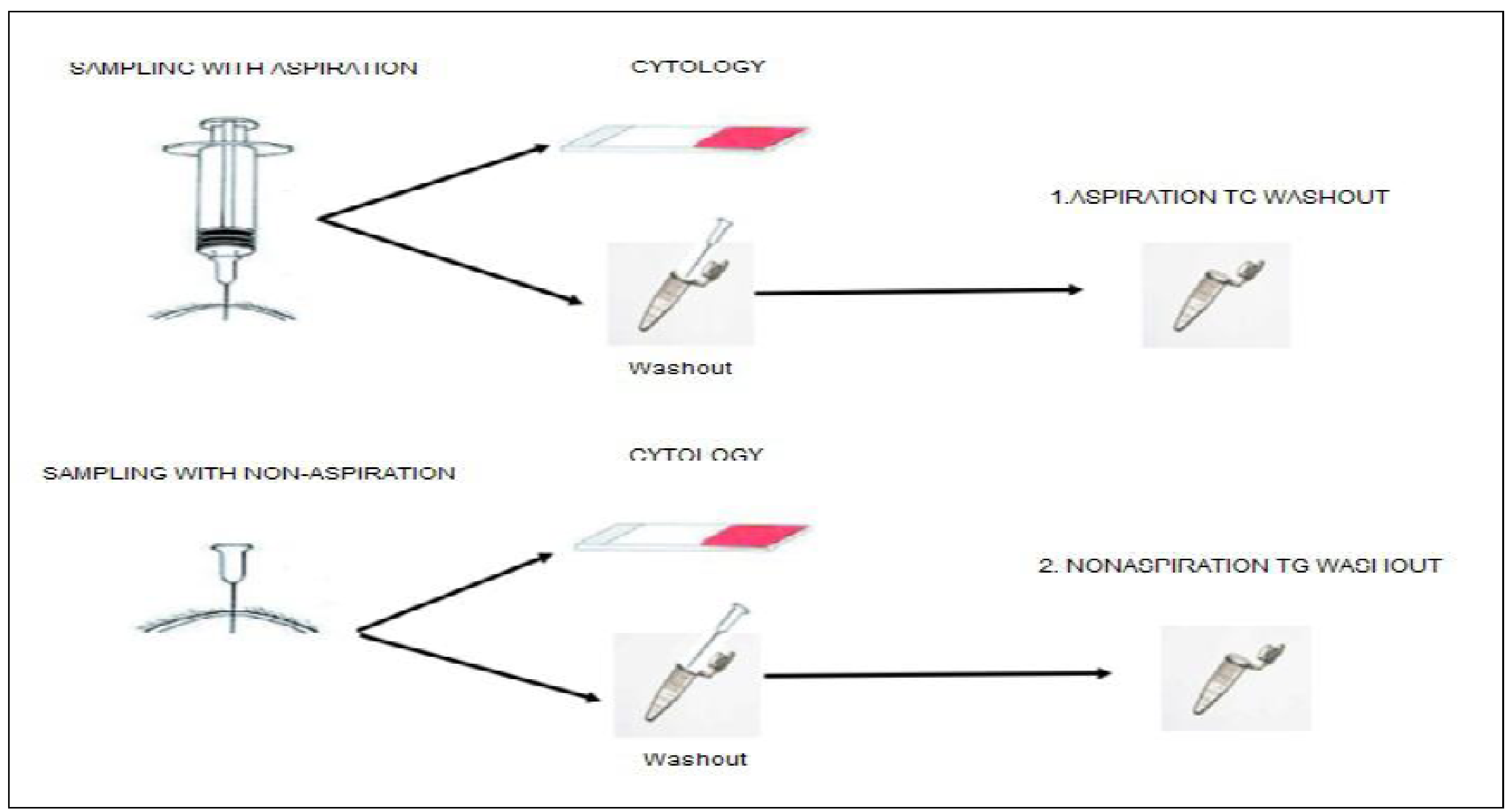

B) From venous whole blood and serum

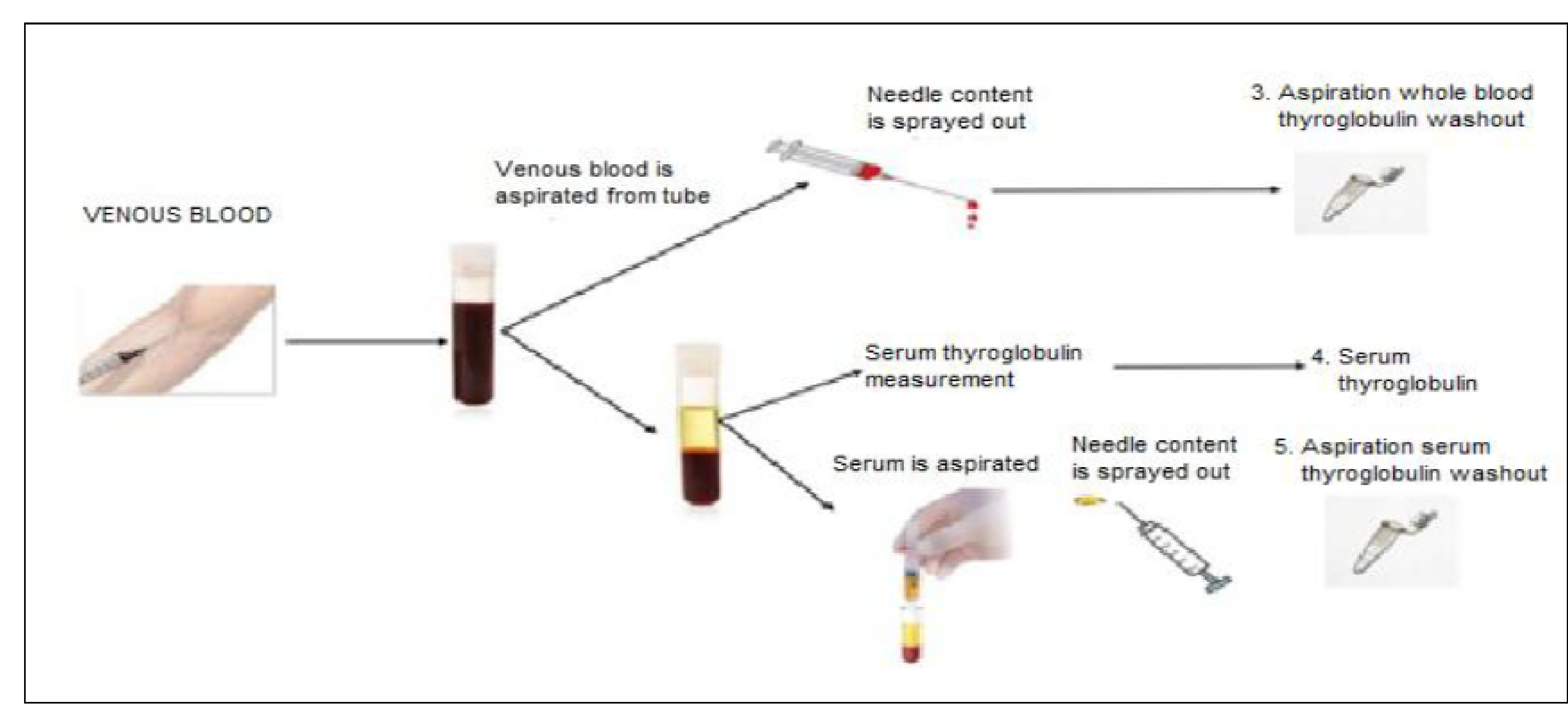

Table 1: Diagnostic performance of methods to differentiate benign and malignant lymph nodes at the best cut-off values

\begin{tabular}{lccc}
\hline Variables & Cut-off & Sensitivity & Specifity \\
\hline FNNA-Tg & $>15,86$ & $71 \%$ & $100 \%$ \\
FNB-Tg & $>31,80$ & $71 \%$ & $100 \%$ \\
FNNA-Tg/Whole blood washout-Tg & $>5.33$ & $100 \%$ & $100 \%$ \\
FNNA-Tg/Serum washout-Tg & $>3,28$ & $100 \%$ & $100 \%$ \\
FNB-Tg/Whole blood washout -Tg & $>5.44$ & $100 \%$ & $100 \%$ \\
FNB-Tg/Serum washout -Tg & $>3,95$ & $100 \%$ & $100 \%$ \\
\hline
\end{tabular}

\section{Conclusion:}

Determining ratio of FNB-Tg to whole blood washout-Tg and/or serum washout-Tg might be a promising method for increasing accuracy and providing standardization of lymph node aspiration to detect malignant lymph nodes. 